\title{
Formação de acadêmicos de enfermagem para o cuidado da saúde mental de crianças e adolescentes
}

\author{
Training of nursing academics for children's mental health care
}

Formación académica de enfermería para la atención de salud mental de los niños

Nara Karoliny Carvalho do Monte Sá1*, Ivanildo Gonçalves Costa Junior ${ }^{1}$, José Wilian de Carvalho ${ }^{1}$, Delmo de Carvalho Alencar², Lany Leide de Castro Rocha Campelo ${ }^{1}$.

\section{RESUMO}

Objetivo: Identificar como o cuidado da saúde mental de crianças e adolescentes é abordado no currículo de graduação em Enfermagem e conhecer a experiência acadêmica e extra acadêmica de graduandos de enfermagem quanto ao cuidado da saúde mental infanto-juvenil. Métodos: Trata-se de estudo descritivo, realizado em duas etapas, documental e quali-quantitativa, em uma instituição pública de ensino superior na cidade de Picos, Piauí, Brasil, no período de março a abril de 2019. Resultados: Verificou-se que as disciplinas Enfermagem em Saúde Mental e Saúde da criança e do adolescente dão ênfase respectivamente ao "Cuidado em Saúde Mental" e "Saúde da Criança e do Adolescente" de forma isolada. Os acadêmicos, em sua maioria, apesar de identificarem patologias de ordem mental e reconhecerem a importância da Enfermagem nesta área, admitiram não estar aptos a prestarem o devido cuidado a esta população, tendo em vista que durante a graduação não existe um foco no ensino da temática, deixando lacunas na formação dos profissionais enfermeiros da instituição. Conclusão: Faz-se necessário revisão do projeto político pedagógico para incluir o cuidado da saúde mental infanto-juvenil na grade curricular do curso, visando melhor formação dos enfermeiros para esta demanda crescente de cuidados.

Palavras-chave: Educação em enfermagem, Cuidados de enfermagem, Saúde mental, Criança, Adolescente.

\section{ABSTRACT}

Objective: To identify how the mental health care of children and adolescents is addressed in the undergraduate nursing curriculum and to know the academic and extra-academic experience of nursing students regarding the care of children and adolescents' mental health. Methods: This is a descriptive study, carried out in two stages, documentary and qualitative and quantitative, in a public institution of higher education in the city of Picos, Piauí, Brazil, from March to April 2019. Results: It was found that the disciplines Nursing in Mental Health and Health of children and adolescents place emphasis on "Mental Health Care" and "Child and Adolescent Health" respectively. Most academics, despite identifying mental disorders and recognizing the importance of nursing in this area, admitted that they were not able to provide due care to this population, considering that during graduation there is no focus on teaching thematic, leaving gaps in the training of the institution's professional nurses. Conclusion: It is necessary to revise the pedagogical political project to include child and adolescent mental health care in the course curriculum, aiming at better training of nurses for this growing demand for care.

Key words: Nursing education, Nursing care, Mental health, Child, Adolescent.

1 Universidade Federal do Piauí (UFPI), Picos - PI. *E-mail: narakaroliny@hotmail.com

${ }^{2}$ Fundação Oswaldo Cruz (FIOCRUZ), Teresina - PI.

SUBMETIDO EM: 02/2020

ACEITO EM: 2/2020

PUBLICADO EM: 3/2020 


\section{RESUMEN}

Objetivo: Identificar cómo se aborda la atención de salud mental para niños y adolescentes en el plan de estudios de enfermería de pregrado y conocer la experiencia académica y extra-académica de los estudiantes de enfermería con respecto al cuidado de la salud mental de niños y adolescentes. Métodos: Estudio descriptivo, realizado en dos etapas, documental y cualitativo y cuantitativo, en una institución pública de educación superior en la ciudad de Picos, Piauí, Brasil, de marzo a abril de 2019. Resultados: Se encontró que las disciplinas de Enfermería en Salud Mental y Salud de niños y adolescentes ponen énfasis en "Atención de Salud Mental" y "Salud de Niños y Adolescentes" respectivamente. La mayoría de los académicos, a pesar de identificar los trastornos mentales y reconocer la importancia de la Enfermería en esta área, admitieron que no pudieron brindar la debida atención a esta población, considerando que durante la graduación no se enfoca en la enseñanza temático, dejando brechas en la formación de los profesionales de enfermería de la institución. Conclusión: Es necesario revisar el proyecto político pedagógico para incluir la atención de salud mental de niños y adolescentes en el plan de estudios del curso, con el objetivo de mejorar la capacitación de las enfermeras para esta creciente demanda de atención.

Palabras clave: Educación en enfermería, Atención de enfermería, Salud mental, Niño, Adolescente.

\section{INTRODUÇÃO}

Grande parcela das pessoas que buscam serviços de saúde mental é composta por crianças e adolescentes, em sua maioria do sexo masculino. Entre os diversos motivos que levam seus responsáveis a buscarem ajuda profissional, encontram-se o mau desempenho escolar, comportamento agressivo e desobediência em casa e no ambiente escolar (PACHÊCO MVGM, et al., 2017). Problemas de saúde mental em crianças e adolescentes podem ocorrer por diversos fatores, tais como problemas genéticos, desordens cerebrais, violência, perda de pessoas próximas, adversidades crônicas e fatores estressantes agudos, transtornos no desenvolvimento, processos de adoção, além dos aspectos culturais e sociais que geram impactos significativos no desenvolvimento infantil e na formação da personalidade do adolescente (EBERT M, et al., 2015).

Há quase duas décadas a saúde mental infanto-juvenil foi reconhecida pelas instâncias governamentais brasileiras como uma questão de saúde pública que deve ser integrada ao conjunto de ações do Sistema Único de Saúde (SUS), tornando-se parte integrante da política geral de saúde mental. Antes disso, as ações relacionadas à saúde mental na infância e adolescência no país, eram delegadas aos setores de educação e assistência social, sendo quase ausente a participação de profissionais da área da saúde (ROSSI LM, et al., 2019).

As ações de saúde mental para crianças e adolescentes antes voltadas apenas ao atendimento de pessoas com sofrimento psíquico grave, passaram a incluir situações de risco ou vulnerabilidade social, como tráfico, prostituição, consumo de álcool e outras drogas e violência, assim como problemas relacionados a dificuldades escolares, comportamentos agressivos, automutilação e isolamento social (ASSIS SG, et al., 2009). Diante da variedade de problemas ou situações que podem incidir sobre a saúde mental de crianças e adolescentes, o cuidado prestado deve levar em consideração os tipos de transtornos, os fatores de risco e proteção, bem como a organização dos serviços e estratégias de intervenção específicas para esta população, que possui peculiaridades que a difere da população adulta (ROSSI LM, et al., 2019).

Desde o movimento da Reforma Psiquiátrica e implementação da política de saúde mental infanto-juvenil como pauta a ser atendida pelo SUS, tem havido progressos no que se refere ao cuidado da saúde mental de crianças e adolescentes, onde se destaca a criação dos Centros de Atenção Psicossocial Infanto-juvenis (CAPSi), regulamentados em 2002 pela Portaria 336 do Ministério da Saúde, destinados ao acolhimento e tratamento de crianças e adolescentes com problemas mentais (BRAGA CP e d'OLIVEIRA AFPL, 2019). Apesar dos investimentos em equipamentos assistenciais referentes à implantação de estratégias em saúde 
mental infanto-juvenil em atendimento aos direitos garantidos pelo Estatuto da Criança e do Adolescente (ECA), a temática voltada à atenção à saúde mental desta população não é abordada com tanta intensidade e frequência em artigos e textos acadêmicos, documentos oficiais ou relatórios técnicos do Ministério da Saúde (PEREIRA MO, et al., 2014). Isso pode refletir ou ser reflexo da pouca abordagem da temática na formação acadêmica de profissionais da saúde, em especial da enfermagem (MONTEIRO ARM, 2003; RODRIGUES J, et al., 2012).

Metodologicamente falando, no ensino da saúde mental atual, seja em sala de aula ou campos de estágios, persistem várias divergências de interesses e um ensino com ênfase nas psicopatologias, que ainda não abrangem os preceitos da Reforma Psiquiátrica, estando centrados na instituição psiquiátrica e reforçando saberes e práticas de exclusão de doenças da mente. Com isso, surgem problemas no reconhecimento, tratamento adequado, preconceito e segregação dos pacientes, se opondo ao discurso pregado por profissionais e instituições de ensino acadêmico, refletindo também na escassa produção científica sobre a saúde mental geral (VILLELA JC, et al., 2013).

Considerando a necessidade de formar enfermeiros com habilidades e competências para atender as demandas de saúde mental infanto-juvenis, faz-se importante conhecer a realidade do curso de Enfermagem de uma Instituição de Ensino Superior, investigando como este tema é abordado na grade curricular. Para tanto, o estudo teve como objetivos identificar como o cuidado da saúde mental de crianças e adolescentes é abordado no currículo de Enfermagem de uma universidade e conhecer a experiência de graduandos de enfermagem quanto ao cuidado da saúde mental infanto-juvenil.

\section{MÉTODOS}

Trata-se de estudo descritivo, realizado em duas etapas, documental e quali-quantitativa, desenvolvido em uma Instituição Pública de Ensino Superior localizada no município de Picos, Piauí, Brasil. Participaram do estudo 96 graduandos de Enfermagem. A coleta de dados foi realizada no período de março a abril de 2019. A primeira etapa, documental, objetivou identificar as disciplinas e períodos que abordassem os conteúdos: cuidado da criança e do adolescente e cuidado de enfermagem em saúde mental. Esta etapa do estudo contou com a busca e identificação de conteúdos relacionados ao cuidado da saúde mental de crianças e adolescentes nos planos de disciplina de cada período do curso de enfermagem.

A busca considerou a descrição dos conteúdos abordados em aulas teóricas e práticas, além das atividades propostas, tais como seminários envolvendo o tema, visitas a instituições que prestassem cuidados a crianças/adolescentes em sofrimento mental, filmes e leituras propostas, bem como a literatura recomendada. Foram considerados todos os planos de disciplina a partir do ano de ingresso dos alunos até o último período do curso. Para identificação das informações pertinentes ao estudo a partir da leitura dos documentos (planos de disciplina) foi utilizado um instrumento elaborado para este fim.

A segunda etapa, quali-quantitativa, com aplicação de questionário aos graduandos dos períodos em que foram identificadas a presença de disciplinas que abordaram os conteúdos descritos na etapa documental, objetivou obter informações sociodemográficas dos sujeitos e o seu conhecimento sobre aspectos relacionados à saúde mental de crianças e adolescentes considerando as disciplinas cursadas e experiências extra acadêmicas. O critério de inclusão referente à segunda etapa do estudo foi: estar devidamente matriculado no curso de bacharelado em Enfermagem da instituição. Foram excluídos acadêmicos que não estavam frequentando as aulas ou que estavam de atestado médico no período da coleta de dados. Os dados coletados do estudo documental foram tabulados e analisados descritivamente conforme período, disciplina/ano e método de abordagem da temática. Os dados sociodemográficos e demais informações referentes ao questionário utilizados na segunda etapa foram analisados conforme estatística descritiva.

Todos os participantes da pesquisa foram informados acerca do objetivo do estudo e dos preceitos éticos que o orientam, descritos na Resolução no 466/2012, do Conselho Nacional de Saúde, e assinaram o Termo de Consentimento Livre e Esclarecido (TCLE). Este estudo foi aprovado pelo Comitê de Ética em Pesquisa (CEP) da Universidade Federal do Piauí, sob Parecer n³.207.760, de 19 de março de 2019. 


\section{RESULTADOS}

Verificou-se que conteúdos relacionados aos temas "Saúde da Criança e do Adolescente" e "Saúde Mental", são abordados ao longo do curso de Bacharelado em Enfermagem durante o terceiro, sexto, oitavo e nono semestres. No terceiro semestre, os conteúdos relacionados à temática são abordados na disciplina Saúde Mental; no sexto, na disciplina Saúde da Criança e do Adolescente; no oitavo e nono semestres da graduação, durante o estágio nas Unidades Básicas de Saúde (UBS) e nos dois Centros de Atenção Psicossocial do município durantes os estágios curriculares I e II. Embora não conste na ementa dos componentes curriculares, durante o estágio nas UBS, os graduandos têmoportunidade de ter contato com a população infanto juvenil durante a consulta de enfermagem e puericultura, além de participarem e promoverem ações de educação em saúde. Nos CAPS, o contato é direcionado à população de adolescentes e adultos usuários dos serviços com transtornos mentais.

Pôde-se perceber que a temática saúde mental e saúde da criança permeia quase metade do número total de períodos que compõem o curso, no entanto conforme o instrumento elaborado para análise dos planos de ensino, é possível observar que nenhuma das disciplinas traz um objetivo geral ou específico acerca da temática "cuidado em saúde mental de crianças e adolescentes", assim, quando se trata do conteúdo programático, os temas saúde mental e saúde da criança e do adolescente são abordados sem um foco específico na temática de saúde mental do público infanto juvenil. (Quadro 1)

Observou-se que as referências básicas e complementares do plano de ensino de algumas disciplinas possibilitam a abordagem ou explanação de problemas relacionados à saúde mental de crianças e adolescentes, porém o conteúdo não é abordado em sala de aula. Na disciplina Enfermagem em Saúde Mental, o tema cuidado em saúde mental infanto-juvenil não aparece na ementa, nem tampouco nos objetivos. Na disciplina Saúde da criança e do adolescente, a ementa cita a atenção à saúde mental, no entanto, em seus objetivos não há nada relacionado ao tema.

O plano de ensino da disciplina Estágio curricular I não especifica em sua ementa ou objetivos, conteúdos voltados para a temática trabalhada neste estudo. A disciplina Estágio curricular II não inclui a temática na ementa e nos objetivos. As aulas práticas das disciplinas Saúde da criança e do adolescente, e Enfermagem em saúde mental e os estágios do componente Estágio Curricular I e II nos diversos serviços de saúde da cidade oportunizam, contudo não asseguram que os graduandos tenham um contato próximo com crianças e adolescentes com problemas de saúde mental. 


\section{Revista Eletrônica Acervo Saúde / Electronic Journal Collection Health ｜ ISSN 2178-2091}

Quadro 1 - Abordagem da temática nos planos de ensino do curso de Bacharelado em Enfermagem. Picos, Piauí, 2019.

\begin{tabular}{|c|c|c|c|c|c|c|}
\hline Disciplina & Período & Ementa & $\begin{array}{l}\text { Objetivos } \\
\text { Gerais ou } \\
\text { Específicos }\end{array}$ & $\begin{array}{c}\text { Conteúdo } \\
\text { programático }\end{array}$ & $\begin{array}{l}\text { Estratégias de } \\
\text { abordagem }\end{array}$ & Referências \\
\hline $\begin{array}{l}\text { Enfermagem em } \\
\text { Saúde Mental }\end{array}$ & $3^{\circ}$ & - & - & $\begin{array}{l}\text { Dispositivos de atenção à } \\
\text { saúde mental no Brasil: a } \\
\text { Rede de Atenção } \\
\text { Psicossocial (RAPS) } \\
\text { (incluindo o CAPSi) }\end{array}$ & Aulas teóricas & $\begin{array}{l}\text { Ministério da Saúde. } \\
\text { Portaria no } 3.088 \text { de } 23 \\
\text { de dezembro de } 2011 .\end{array}$ \\
\hline $\begin{array}{c}\text { Saúde da Criança e } \\
\text { do Adolescente }\end{array}$ & $6^{\circ}$ & $\begin{array}{c}\text { Saúde mental da criança } \\
\text { e do adolescente }\end{array}$ & - & $\begin{array}{c}\text { Cuidados de enfermagem } \\
\text { relacionados à consulta de } \\
\text { puericultura. }\end{array}$ & - & $\begin{array}{c}\text { SANDSTROM, C.I. A } \\
\text { psicologia da infância e } \\
\text { da adolescência. Rio } \\
\text { de Janeiro: Zahar, } \\
1997 .\end{array}$ \\
\hline Estágio Curricular I & $8^{\circ}$ & - & - & $\begin{array}{c}\text { Realizar consulta de } \\
\text { enfermagem de acordo com } \\
\text { os programas de atenção à } \\
\text { saúde, estabelecidos pelo } \\
\text { Ministério da Saúde (Saúde } \\
\text { da Criança) }\end{array}$ & Estágios & - \\
\hline Estágio Curricular II & $9^{\circ}$ & - & - & $\begin{array}{c}\text { Realizar a assistência de } \\
\text { enfermagem a pacientes } \\
\text { portadores de transtornos } \\
\text { mentais em } \\
\text { acompanhamento no Centro } \\
\text { de Atenção Psicossocial II } \\
\text { (CAPS II) }\end{array}$ & Estágios & - \\
\hline
\end{tabular}

Fonte: Sá NKCM, et al., 2020. 
Participaram da segunda etapa do estudo, 96 dos 121 graduandos de quatro períodos do curso de bacharelado em enfermagem em que foram identificados a presença de disciplinas que abordassem os conteúdos descritos na etapa documental.

Quanto à caracterização sociodemográfica dos graduandos, verificou-se que a maioria $65(67,7 \%)$ eram do sexo feminino, apresentando uma frequência maior de pardos $52(54,7 \%)$. A maioria dos respondentes desta etapa cursava entre o $7^{\circ}$ e $9^{\circ}$ semestres $61(64,2 \%)$ do curso de graduação. (Tabela 1).

Tabela 1 - Perfil sociodemográfico dos acadêmicos de Enfermagem. Picos, Piauí, 2019.

\begin{tabular}{lllll}
\hline & Variáveis & $\mathbf{n}$ & $\%^{*}$ & Média \pm DP** \\
\hline Idade & & & & $22,73 \pm 3,91$ \\
\hline Sexo & & & & \\
\hline & Feminino & 65 & 67,7 & \\
& Masculino & 31 & 32,3 & \\
\hline
\end{tabular}

\section{Cor/Raça}

\begin{tabular}{|c|c|c|c|}
\hline Branco(a) & 32 & 33,7 & \\
\hline Pardo(a) & 52 & 54,7 & \\
\hline Negro(a) & 10 & 10,5 & \\
\hline Amarelo(a) & 01 & 1,1 & \\
\hline Período & & & $7,14 \pm 2,09$ \\
\hline $1^{\circ}$ ao $3^{\circ}$ semestres & 15 & 15,8 & \\
\hline $4^{\circ}$ ao $6^{\circ}$ semestres & 19 & 20,0 & \\
\hline $7^{\circ}$ ao $9^{\circ}$ semestres & 61 & 64,2 & \\
\hline
\end{tabular}

\footnotetext{
Legenda:

* Percentual válido.

** DP: desvio-padrão.
}

Fonte: Sá NKCM, et al., 2020.

No que diz respeito à análise da formação do acadêmico de Enfermagem quanto ao cuidado em Saúde Mental de crianças e adolescentes, foi possível observar que a maioria dos graduandos 84 (86,5\%) relataram não conviver com alguma criança/adolescente com problema de saúde mental. Um total de 43 $(63,2 \%)$ acadêmicos consideraram-se incapazes de identificar o tipo de transtorno de saúde mental que crianças/adolescentes apresentam. (Tabela 2). 
Tabela 2 - Formação do acadêmico de Enfermagem quanto ao cuidado em saúde mental de crianças e adolescentes. Picos, Piauí, 2019.

\begin{tabular}{lcc}
\hline \multicolumn{1}{c}{ Variáveis } & $\mathbf{n}$ & $\%^{*}$ \\
\hline Convive com alguma criança / adolescente com problema de saúde mental? & 13 & 13,5 \\
Sim & 84 & 86,5 \\
Não & \\
\hline Você é capaz de identificar que tipo de problema de saúde mental a criança / & 25 & 36,8 \\
adolescente apresenta? & 43 & 63,2 \\
\hline Sim & \\
Não & 51 \\
\hline No decorrer do curso, você vivenciou alguma experiência teórica ou prática sobre o & 54,3 \\
cuidado de enfermagem a criança / adolescente com problemas de saúde mental? & 43 & 45,7 \\
Sim & \\
Não & 19 \\
\hline Considerando seu período de formação, você se considera capaz ou apto a realizar & 20,2 \\
assistência de enfermagem a criança / adolescente com problemas de saúde mental? & 75 & 79,8 \\
Sim & \\
Não & 11 \\
\hline Possui alguma vivência ou experiência extra acadêmica no cuidado de criança / & 11,8 \\
adolescente com problemas de saúde mental? & 82 & 88,2 \\
\hline Sim & & \\
Não & 92 & 97,9 \\
\hline Você acredita que cuidar da saúde mental faz parte do papel do enfermeiro? & 2,1 \\
\hline Sim & 2
\end{tabular}

Legenda: * Percentual válido

Fonte: Sá NKCM, et al., 2020.

Para alguns alunos, esta limitação decorre da inexperiência relacionada ao fato de ainda não terem concluído o curso de graduação ou ainda por esta temática não ter sido abordada em sala de aula, aulas práticas ou estágios.

Em meu atual período de formação ainda não considero possuir as habilidades necessárias para realizar uma assistência de enfermagem a crianças/adolescentes com problemas de saúde mental, de forma eficaz. (Q05)

Não sei, porque ainda estou no início da disciplina. (Q69)

Não vi durante o curso, práticas ou aulas que abordassem. (Q43)

Durante a disciplina de saúde mental não é dado o devido suporte para os alunos para possibilitar a assistência a crianças e adolescentes com problemas de saúde mental, não só na disciplina como também durante toda a graduação. (Q32)

Quando questionados sobre terem vivenciado ao longo do curso, alguma experiência teórica ou prática sobre o cuidado de enfermagem à criança/adolescente com problemas de saúde mental, 51 (54,3\%) responderam que sim. Um total de $82(88,2 \%)$ dos acadêmicos afirmaram possuir alguma vivência ou experiência extra acadêmica no cuidado de criança/adolescente com problemas de saúde mental. Quando questionados sobre o cuidar em saúde mental ser papel do enfermeiro, as respostas foram majoritariamente que sim, representando um total de 92 (97,9\%), como mostram os dados da (Tabela 2).

Os graduandos de enfermagem reconheceram que crianças e adolescentes com problemas de saúde mental são alvo de cuidado do Sistema Único de Saúde brasileiro, bem como dos enfermeiros, como profissionais que o constituem e que devem estar habilitados para acompanhar e cuidar também dos aspectos relacionados ao desenvolvimento emocional de crianças e adolescentes, assistindo a este público em todas as suas necessidades a fim de proporcionar-lhes melhor qualidade de vida. 
Pois também faz parte da população e de acordo com o SUS não podemos fazer distinção a nenhuma pessoa. (Q19)

Porque ele (o profissional enfermeiro) atua em todas as áreas da atenção à saúde. (Q76)

O trabalho em saúde mental deve atender as necessidades específicas das crianças, deve-se fazer um acompanhamento do desenvolvimento físico e mental deste. (Q21)

Pois o âmbito da saúde mental entra no cuidar, pois há necessidade. (Q89)

Ao cuidar da saúde mental deve ser feito de forma integrada, fazendo o enfermeiro ponte dessa atuação/atenção integral. (Q12)

É uma área que deve dar uma atenção maior e o enfermeiro pode ajudar de diversas formas desde o amparo psicológico até medidas de cuidados aos enfermos. (Q56)

A enfermagem pode proporcionar uma melhoria na qualidade de vida do paciente. (Q91)

O cuidado prestado ao indivíduo que apresenta problemas mentais deve ser feito por um enfermeiro, desde que este seja capacitado para exercer tal função. (Q02)

Quanto ao tipo de proximidade que o acadêmico possui em relação à convivência com a criança/adolescente que possui problema de saúde mental, $53,8 \%$ relataram ser por meio familiar. (Gráfico 1)

Gráfico 1 - Distribuição das respostas sobre o tipo de proximidade com a criança/adolescente que possui problema de saúde mental, com a qual o acadêmico de enfermagem convive. Picos, Piauí, 2019.

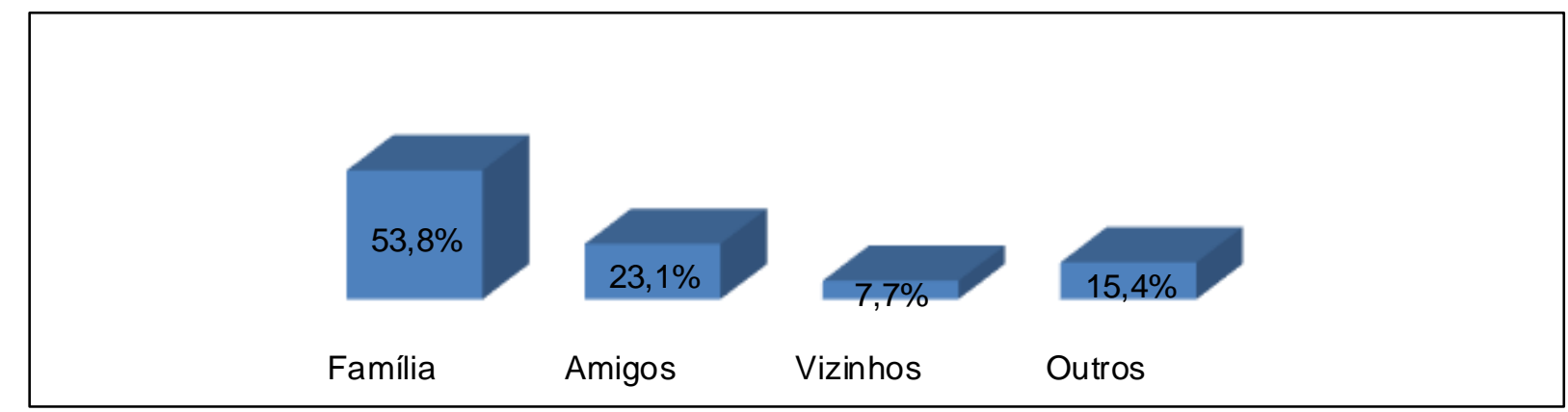

Legenda: * Percentual válido de acordo com o número de acadêmicos que responderam conviver com alguma criança/adolescente com problema de saúde mental.

Fonte: Sá NKCM, et al., 2020.

Destaca-se que 13 dos 96 alunos admitiram conviver em certo grau de proximidade com crianças e/ou adolescentes com problemas de saúde mental, contato decorrente de um relacionamento familiar, comunitário ou da sua prática como acadêmico de enfermagem:

Vivenciei em uma sala de aula com um aluno especial. (Q23)

Prima apresenta atraso no desenvolvimento cognitivo. (Q54)

Com primos que possuem autismo. (Q65)

Em casa e na escola. (Q19) 
O maior contato com o tema saúde mental de crianças e adolescentes se deu a partir de aulas teóricas, representando $70,6 \%$ das respostas seguido de aulas práticas com $40,9 \%$ das respostas. (Gráfico 2).

Gráfico 2 - Distribuição das respostas sobre o tipo de experiência teórica ou prática que o acadêmico vivenciou sobre o cuidado de enfermagem a crianças/ adolescentes com problemas de saúde mental. Picos, Piauí, 2019.

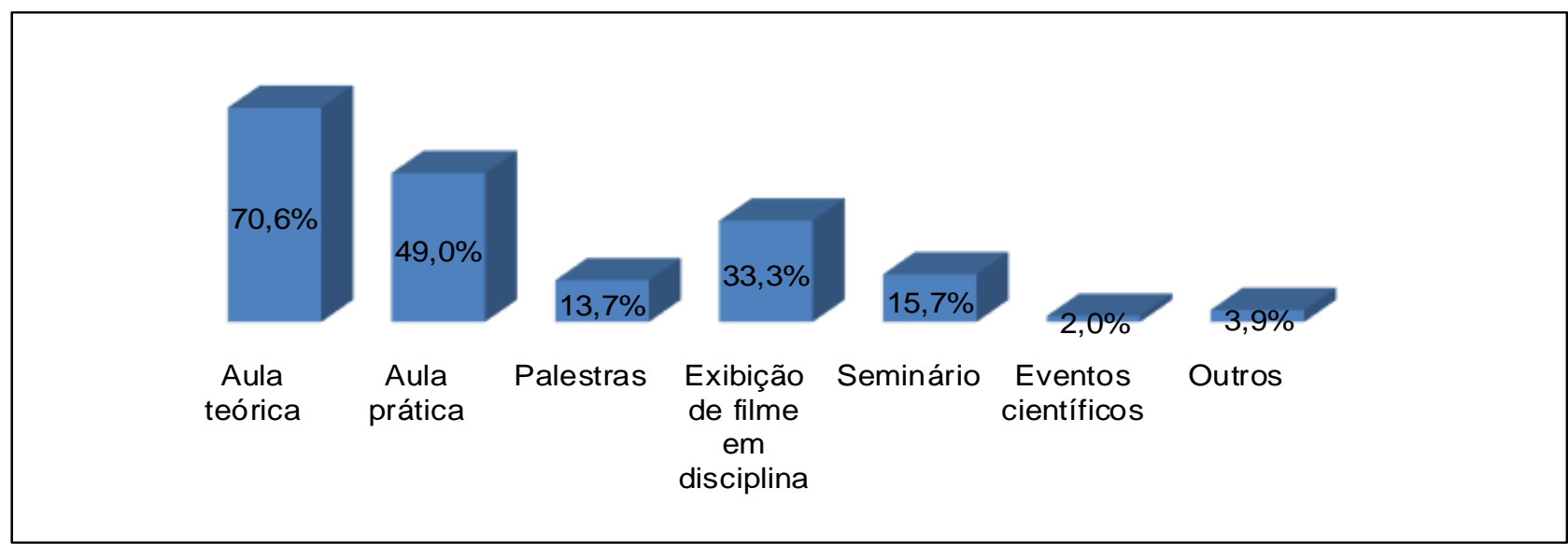

Legenda: *Percentual válido de acordo com o número de acadêmicos que responderam ter vivenciado alguma experiência teórica ou prática sobre o cuidado de enfermagem a crianças/adolescentes com problemas de saúde mental.

Fonte: Sá NKCM, et al., 2020.

Dos que responderam ser capazes de identificar que tipo de problema de saúde mental a criança/adolescente apresenta, sete $(28,0 \%)$ citaram reconhecer a ansiedade, sete $(28,0 \%)$ autismo, três $(12,0 \%)$ síndrome de Down, três $(12,0 \%)$ depressão, três $(12,0 \%)$ esquizofrenia, dois $(8,0 \%)$ Transtorno Obsessivo Compulsivo, um (4,0\%) transtorno afetivo bipolar, um (4,0\%) síndrome de borderline, um (4,0\%) déficit de atenção e um (4,0\%) atraso no desenvolvimento. (Gráfico 3)

Gráfico 3 - Distribuição das respostas sobre o tipo de problema de saúde mental da criança/adolescente que o acadêmico de enfermagem é capaz de identificar. Picos, Piauí, 2019.

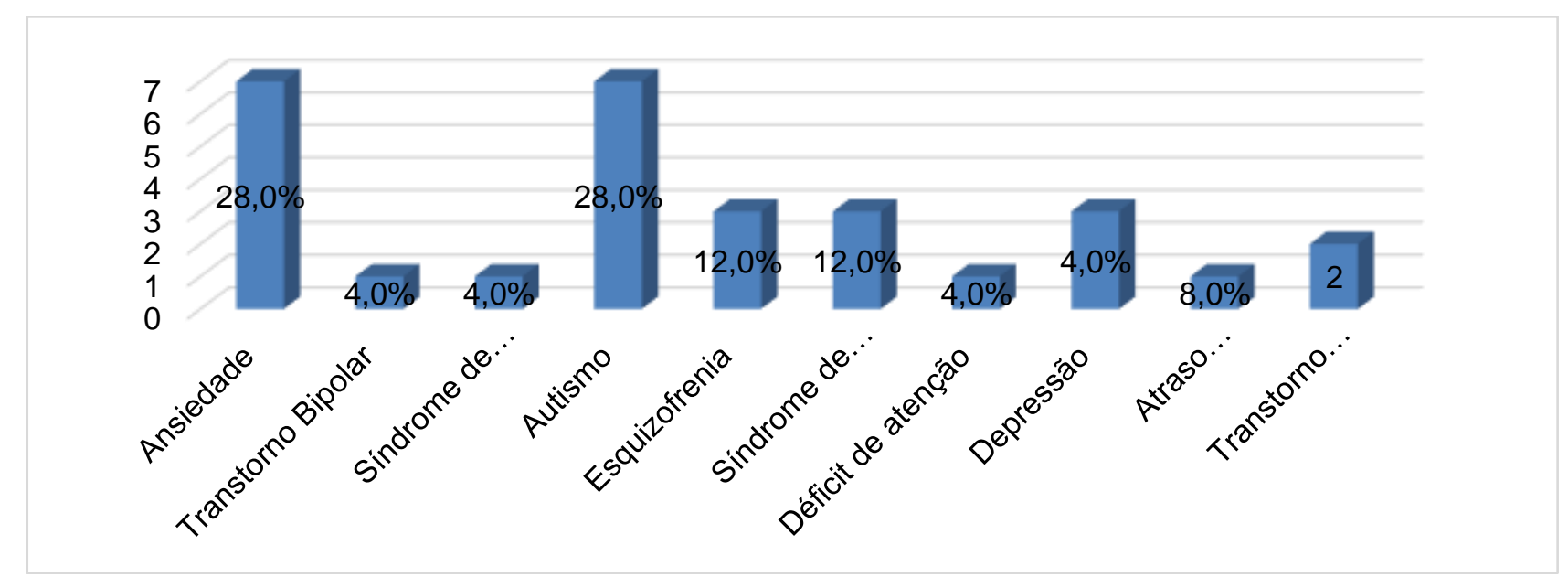

Legenda: * Percentual válido de acordo com o número de acadêmicos que responderam ser capazes de identificar o tipo de problema de saúde mental que a criança/adolescente apresenta.

Fonte: Sá NKCM, et al., 2020. 


\section{DISCUSSÃO}

Atualmente, percebe-se o descompasso entre o ensino e a prática da enfermagem em saúde mental infanto-juvenil e desses com as políticas nacionais de saúde mental. Este cenário é um dos fatores que colabora para a formação de profissionais acríticos e pouco atuantes politicamente dentro de um contexto de Reforma Psiquiátrica (RODRIGUES J, et al., 2012; VILLELA JC, et al., 2013; EVANGELISTA DL e IVO OP, 2014).

Os dados vão ao encontro dos achados de outro estudo que chama atenção para o fato de que profissionais da atenção básica, incluindo enfermeiros, relatam insegurança na identificação de problemas de saúde mental em crianças e adolescentes, usando como argumento a falta de um "olhar diferenciado" para identificar questões inerentes ao desenvolvimento infanto juvenil, daquelas que exigem a necessidade de intervenção, o que gera incertezas diante de casos de problemas de saúde mental, levando a recorrente referenciamento desnecessário do sujeito para serviços especializados (TEIXEIRA MR, et al., 2017).

Outro estudo evidencia que apesar de os profissionais enfermeiros estarem inseridos no atendimento a indivíduos com problemas mentais, os mesmos nem sempre são preparados para atuar na área de saúde mental, deixando claro que as instituições de ensino deixam a desejar quando se trata da abordagem a esta temática, colocando profissionais no mercado de trabalho sem muito conhecimento e confiança no cuidado realizado (CAVALCANTI PCS, et al., 2014).

O ensino de enfermagem em saúde mental de crianças e adolescentes deve dar condições para que o graduando desenvolva habilidades científicas, humanísticas e técnicas, conhecimento com especificidade na área em questão, que o instrumentalize para sua prática profissional. Contudo, estudostêm demonstrado a existência de dificuldades em adequar o conteúdo teórico-prático à realidade assistencial, que em muitos casos, ainda se mantém deficitária de pessoal qualificado (CAVALCANTI PCS, et al., 2014; GONÇALVES AM, et al., 2016; TEIXEIRA MR, et al., 2017).

Faz-se necessário que o enfermeiro tenha entendimento de todo o processo terapêutico, sendo o cuidado de enfermagem uma proposta de intervenção terapêutica que favorece a reabilitação psicossocial e o fortalecimento do sujeito com problemas mentais (GONÇALVES AM, et al., 2016). Gonçalves AM, et al. (2016) afirmam ainda, ser privativo do enfermeiro a elaboração de um projeto de intervenções de enfermagem com o objetivo de melhorar o resultado do paciente diante da terapêutica empregada, exigindo que o profissional tenha conhecimentos clínicos acerca da temática.

$O$ atendimento de crianças e adolescentes no CAPS Infantil também é de competência do enfermeiro, sendo seu papel acolher e prestar cuidado de qualidade à criança em sofrimento psíquico, atuando no intuito de reinserir a criança na família e na sociedade, usando estratégias que busquem oferecer o apoio necessário em busca de autonomia e independência da criança ou adolescente (CAVALCANTI PCS, et al., 2014). Essa informação reforça a importância que deve ser dada à abordagem do cuidado da saúde mental da criança e adolescente na formação profissional do enfermeiro, haja vista que, além deste cuidado ser também de sua competência, este é um problema comum no seu cotidiano, principalmente levando em consideração que a saúde mental infanto-juvenil atinge todas as áreas do desenvolvimento, impactando a saúde física e mental também das famílias e o desempenho da criança ou adolescente na escola, gerando consequências que perduram até a vida adulta (DALTRO MCSL, et al., 2018).

As dificuldades mais frequentes na formação de acadêmicos de enfermagem estão relacionadas ao cuidado a pessoas que apresentam algum tipo de transtorno mental, especialmente o público infantojuvenil. Diante disso, considera-se importante que a formação do enfermeiro não seja marcada apenas pela teoria, a fim de proporcionar ao acadêmico uma oportunidade de expandir e aprimorar seus conhecimentos acerca da temática em campos de prática para que tenham oportunidade decontato com todos os ciclos de vida, da infância até a fase adulta (EVANGELISTA DL e IVO OP, 2014). A Resolução CNE/CES no 3, de 7 de novembro de 2001, inclui na grade curricular o Estágio Supervisionado nos dois últimos semestres de curso, a fim de possibilitar ao acadêmico se autodescobrir como profissional, além de proporcionar vivência de habilidades como responsabilidades e liderança de equipe, sendo importante incluir nesta fase da 
formação, estágios em serviços que ofereçam tratamento e acompanhamento a crianças e adolescentes com problemas de saúde mental (BRASIL, 2001). Conhecer o perfil epidemiológico dos transtornos mentais que acometem crianças e adolescentes, além de saber diferenciar cada um quanto aos principais sinais e sintomas, é de suma importância para que seja realizada a correta identificação e encaminhamento deste público para serviços especializados, contribuindo para a organização e planejamento de um atendimento de qualidade aos usuários (THIENGO DL, et al., 2014).

Os agravos na saúde mental de crianças e adolescentes mais frequentes são a depressão, os transtornos de ansiedade, TDAH, transtorno por uso de substâncias, e transtorno de conduta (CARNEIRO ES, et al., 2018). As respostas dos participantes da pesquisa mostraram que por mais que o ensino da saúde mental não seja totalmente satisfatório, os mesmos conseguem associar as vivências do dia a dia com os conhecimentos adquiridos fora da universidade para identificar alguns dos transtornos mentais que acometem crianças e adolescentes, o que pode favorecer o cuidado de enfermagem e tomada de medidas necessárias, como encaminhamento para serviços especializados, quando for o caso.

Foram limitações para a realização do estudo: a falta de publicações e estudos recentes sobre o tema e a recusa de alguns acadêmicos em participar da pesquisa, principalmente das entrevistas, alegando a falta de conhecimento sobre o tema e o medo de não responderem adequadamente aos questionamentos que seriam feitos. A existência de poucos estudos publicados sobre a formação de graduandos de enfermagem na saúde mental de crianças e adolescentes, dificultou a comparação com os achados deste estudo e evidenciou a importância de pesquisas sobre o tema.

\section{CONCLUSÃO}

Foi possível constatar que na grade curricular do curso de Enfermagem da instituição, a disciplina "Enfermagem em saúde mental" aborda predominantemente o cuidado da saúde mental do público adulto, e a disciplina "Saúde da criança e do adolescente" dá ênfase à saúde infanto-juvenil de forma isolada, sem abordagem da saúde mental destes, deixando lacunas na formação dos profissionais enfermeiros da instituição, considerando que durante a graduação não existe um foco no ensino da temática. Os acadêmicos, em sua maioria, apesar de identificarem patologias de ordem mental e reconhecerem a importância da enfermagem nesta área, sentem-se despreparados para cuidar de crianças e adolescentes com problemas de saúde mental.

\section{REFERÊNCIAS}

1. 1.ASSIS SG, et al. Situação de crianças e adolescentes brasileiros em relação à saúde mental e à violência. Ciênc Saúde Coletiva, 2009; 14(2): 349-361.

2. 2.BRAGA CP, d'OLIVEIRA AFPL. Políticas públicas na atenção à saúde mental de crianças e adolescentes: percurso histórico e caminhos de participação. Ciênc Saúde Coletiva, 2019; 24(2): 401-410.

3. BRASIL. Ministério da Educação. Resolução CNE/CES nํ 3, de 7 de novembro de 2001.

4. CARNEIRO ES, et al. Abordagem da equipe de saúde nos agravos de saúde mental de crianças e adolescentes hospitalizados. Rev Soc Bras Enferm Ped, 2018; 18(1): 7-14.

5. CAVALCANTI PCS, et al. O cuidado de enfermagem nos Centros de Atenção Psicossocial. Cienc Cuid Saude, $2014 ; 13(1): 111-119$.

6. DALTRO MCSL, et al. Cuidadores de crianças e adolescentes com transtornos mentais: mudanças na vida social, familiar e sexual. Saúde Soc, 2018; 27(2): 544-555.

7. EBERT M, et al. Mães de crianças com transtorno autístico: percepções e trajetórias. Rev Gaúcha Enferm, 2015; 36(1): 49-55.

8. EVANGELISTA DL, IVO OP. Contribuições do estágio supervisionado para a formação do profissional de enfermagem: expectativas e desafios. Rev Enferm Contemp, 2014; 3(2): 123-130.

9. GONÇALVES AM, et al. Oficinas terapêuticas: intervenção de enfermagem em um serviço de saúde mental infanto-juvenil. CBSM, 2016; 8(19): 107-115.

10. MONTEIRO ARM. Saúde mental como tema transversal no currículo de enfermagem. Rev Bras Enferm, 2003; 56(4): 420-423. 
11. PACHÊCO MVGM, et al. Caracterização e perfil epidemiológico de um serviço de psiquiatria infantil no Recife. Rev SBPH, 2017; 20(2): 136-152.

12. PEREIRA MO, et al. Um olhar sobre a atenção psicossocial a adolescentes em crise a partir de seus itinerários terapêuticos. Cad Saúde Pública, 2014; 30(10): 2145-2154.

13. RODRIGUES J, et al. Ensino do cuidado de enfermagem em saúde mental na graduação em Enfermagem. Acta Paul Enferm, 2012; 25(6): 844-51.

14. ROSSI LM, et al. Crise e saúde mental na adolescência: a história sob a ótica de quem vive. Cad Saúde Pública, 2019; 35(3): e00125018.

15. TEIXEIRA MR, et al. Atenção básica e cuidado colaborativo na atenção psicossocial de crianças e adolescentes: facilitadores e barreiras. Ciênc Saúde Coletiva, 2017; 22(6): 1933-1942.

16. THIENGO DL, et al. Prevalência de transtornos mentais entre crianças e adolescentes e fatores associados: uma revisão sistemática. J Bras Psiquiatr, 2014; 63(4): 360-72.

17. VILLELA JC, et al. O ensino de saúde mental na graduação de enfermagem: um estudo de caso. Texto Contexto Enferm, 2013; 22(2): 397-406. 